\title{
РЕЗУЛЬТАТЫ ДИНАМИЧЕСКОГО НАБЛЮДЕНИЯ ПАЦИЕНТОВ С ПОСЛЕОПЕРАЦИОННЫМ ЦЕНТРАЛЬНЫМ НЕСАХАРНЫМ ДИАБЕТОМ
}

\author{
Михайлова Д.С., Дзеранова Л.К., Пигарова Е.А., Реброва О.Ю., Рожинская Л.Я., Григорьев А.Ю., Азизян В.Н., \\ Иващенко О.В.
}

ФГБУ «НМИЦ эндокринологии» Минздрава России, Москва

ЦЕЛЬ: изучить особенности течения и прогностические факторы развития центрального несахарного диабета после трансназальной аденомэктомии.

МАТЕРИАЛЫ И МЕТОДЫ: обследовано 152 пациента от 18 до 65 лет (медиана и квартили 40 [31; 52]), которым в условиях НМИЦ эндокринологии в 2010-2011 гг. была проведена трансназальная аденомэктомия по поводу болезни Иценко-Кушинга - БИК $(n=71)$, акромегалии $(n=66)$, гормонально-неактивной аденомы гипофиза $(n=9)$, пролактиномы $(n=4)$, синдрома Нельсона $(n=1)$ и тиреотропиномы $(n=1)$. Пациентам до и после операции проводилось общеклиническое обследование, контроль осмоляльности и электролитов мочи и крови, определение уровня копептина, гормональные анализы крови, МРТ головного мозга. Динамическое наблюдение выполнялось через 6, 12 месяцев и 6 лет после операции, к концу наблюдения выбывание составило 55 пациентов (36\%).

PЕЗУЛЬТАТЫ: к моменту выписки из стационара состояние 34 пациентов $(22,4 \%)$ расценено как послеоперационный центральный несахарный диабет - ЦНД, в ходе динамического наблюдения из них у 16 пациентов нарушения разрешились. Из 25 пациентов (16,4\%) с разрешившимися к моменту выписки нарушениями у 3 развился ЦНД в ходе дальнейшего наблюдения. У 91 пациента на момент выписки водно-электролитных нарушений выявлено не было, однако у 1 впоследствии развился ЦНД, у 8 - тЦНД. Таким образом, с учетом выбывания, к концу наблюдения у 15 пациентов (15,5\%) выявлен ЦНД, у 34 пациентов $(35,1 \%)$ - транзиторный ЦНД. Дебют постоянной формы ЦНД приходился на 5 сутки [1; 9,5], транзиторной - на 1 сутки $[1 ; 4,5]$, а медиана разрешения нарушений — на 30 сутки $[1,5 ; 195]$ послеоперационного периода. На момент выписки у пациентов с ЦНД выявлено резкое снижение уровня копептина крови в динамике (до операции медиана и квартили 8,262 [1,157; 10,3], после - 4,9 [4,9; 5,265], p=0,003), однако при сравнении пациентов с нарушениями и без них статистически значимых отличий выявлено не было. По итогам наблюдения, тЦНД более часто развивался у пациентов с БИК по сравнению с другими нозологиями (ОШ 6,098 $(2,306 ; 16,131))$ и у пациентов с микроаденомой по данным МРТ по сравнению с макроаденомой (ОШ 5,281 (1,969; 14,169)). У пациентов с послеоперационной вторичной надпочечниковой недостаточностью чаще отмечалось развитие тЦНД (ОШ $6,843(2,553 ; 18,342))$, а у пациентов со вторичным гипотиреозом - постоянной формы ЦНД (ОШ 9,778 $(2,054 ; 46,550))$, по сравнению с пациентами без тропных нарушений. Иссечение гипофиза, согласно протоколу операции, приводило к более частому развитию постоянной формы ЦНД (ОШ 4,853 (1,336; 17,626)), чем у пациентов без повреждения гипофиза.

Выводы: после выписки из стационара диагноз постоянной или транзиторной форм ЦНД у пациентов, перенесших трансназальную аденомэктомию, должен уточняться по результатам динамического наблюдения. Дебют ЦНД наиболее часто приходится на 1-5 сутки послеоперационного периода, продолжительность транзиторных расстройств варьирует от 1 суток до нескольких месяцев. У пациентов с БИК, микроаденомой гипофиза и вторичной надпочечниковой недостаточностью более вероятно развитие ТЦНД, тогда как у пациентов со вторичным гипотиреозом и повреждением гипофиза в ходе операции постоянной формы.

КЛЮЧЕВЫЕ СЛОВА: послеоперационный несахарный диабет; трансназальная аденомэктомия; копептин. 